\title{
MEASURING THE PARTICULATE BACKSCATTERING OF INLAND WATERS: A COMPARISON OF TECHNIQUES
}

\author{
Glenn Campbell ${ }^{\mathrm{a}, \mathrm{b} *}$ and Stuart R. Phinn ${ }^{\mathrm{a}}$ \\ ${ }^{a}$ Centre for Spatial Environmental Research, School of Geography, Planning and Environmental Management, \\ University of Queensland, St Lucia, Queensland, Australia 4072 \\ ${ }^{\mathrm{b}}$ Australian Centre for Sustainable Catchments \& Faculty of Engineering and Surveying University of Southern \\ Queensland, Toowoomba, Queensland 4350, Glenn.Campbell@usq.edu.au
}

Commission VII, WG VII/1

KEY WORDS: Scattering Properties; Water Quality; Data Correction; Inherent Optical Properties; Sediment Monitoring

\begin{abstract}
:
The objective of this work was to examine whether the standard particulate backscattering IOP (Inherent Optical Properties) measurement method could be simplified. IOP measurements are essential for parameterising several forms of algorithms used to estimate water quality parameters from airborne and satellite images. Field measurements of the backscattering IOPs are more difficult to make than absorption measurements as correction of the raw Hydroscat-6 backscattering sensor observations is required to allow for the systematic errors associated with the water and water quality parameter absorption. The standard approach involves making simultaneous measurement of the absorption and attenuation of the water with an absorption and attenuation meter ( $a c-9)$ or making assumptions about the particulate backscattering probability. Recently, a number of papers have been published that use an alternative method to retrieve the particulate backscattering spectrum by using laboratory measured absorption values and in situ spectroradiometric observations. The alternative method inverts a model of reflectance iteratively using non-linear least squares fitting to solve for the particulate backscattering at $532 \mathrm{~nm}\left(\mathrm{~b}_{\mathrm{bp} 0}(532)\right)$ and the particulate backscattering spectral slope $(\gamma)$. In this paper, eleven observations made at Burdekin Falls Dam, Australia are used to compare the alternative reflectance method to the conventional corrected Hydroscat- 6 observations. Assessment of the alternative reflectance method showed that the result of the inversions were highly dependent on the starting conditions. To overcome this limitation, Particle Swarm Optimisation, a stochastic search technique which includes a random element in the search approach, was used. It was found that when compared to the conventionally corrected Hydroscat- 6 observations, the alternative reflectance method underestimated $\mathrm{b}_{\mathrm{bp} 0}(532)$ by approximately $50 \%$ and overestimated $\gamma$ by approximately $40 \%$. Differences of this scale in specific backscattering spectra will have a pronounced effect on the parameterisation and subsequent performance of semi-analytical water quality parameter retrieval algorithms. This has implications for any water quality monitoring regimes that rely on semi-analytical algorithms.
\end{abstract}

\section{INTRODUCTION}

The reflectance spectrum of water is a result of the cumulative interactions of light with the water itself and its associated water quality constituents, including chlorophyll and other pigments, tripton (the non-algal particles of the suspended particulate matter), and coloured dissolved organic matter (CDOM). Each of these water quality constituents have inherent optical properties (IOPs), knowledge of which is necessary to retrieve the water quality constituent concentrations from satellite or airborne remotely sensed data or from field spectroradiometers. Field measurement of the backscattering IOPs are more difficult to make than absorption measurements as correction of the raw Hydroscat-6 backscattering sensor observations is required to allow for the systematic errors associated with the water and water quality parameter absorption. The standard approach involves making simultaneous measurement of the absorption and attenuation of the water with an absorption and attenuation meter (ac-9), or making assumptions about the particulate backscattering probability.

Recently a number of papers have been published that use an alternative method to retrieve the particulate backscattering spectrum by using laboratory measured absorption values and in situ spectroradiometric observations (Ma et al. 2009; Wu et al. 2011). In the alternative method, a model of reflectance is inverted iteratively using non-linear least squares fitting to solve for the particulate backscattering at $532 \mathrm{~nm}\left(\mathrm{~b}_{\mathrm{bp} 0}(532)\right)$ and the particulate backscattering spectral slope $(\gamma)$. The work presented in this paper compares the alternative method to the standard particulate backscattering IOP measurement method.

\section{MATERIALS AND METHODS}

\subsection{Study Site}

The Burdekin Falls Dam $\left(20^{\circ} 37^{\prime} \mathrm{S}, 147^{\circ} 0^{\prime} \mathrm{E}\right)$ receives inputs from four major sub-catchments that cover a total area of $114,000 \mathrm{~km}^{2}$. From the north, the Burdekin River has its origin in tropical rainforest but primarily flows through tropical savannah. From the west the lake is fed from the Cape River which rises in reasonably steep sedimentary country and then flows through flat less erodible areas. The Belyando and Suttor Rivers meet just beyond the inundated area and feed the lake from the south. The Belyando River and Suttor River are persistently turbid, with high levels of suspended sediment concentrations. The impoundment is split into an upper and lower basin by a narrow neck of land. Water released from the dam enters the Burdekin River and discharges into the Great Barrier Reef lagoon approximately $200 \mathrm{~km}$ downstream. 


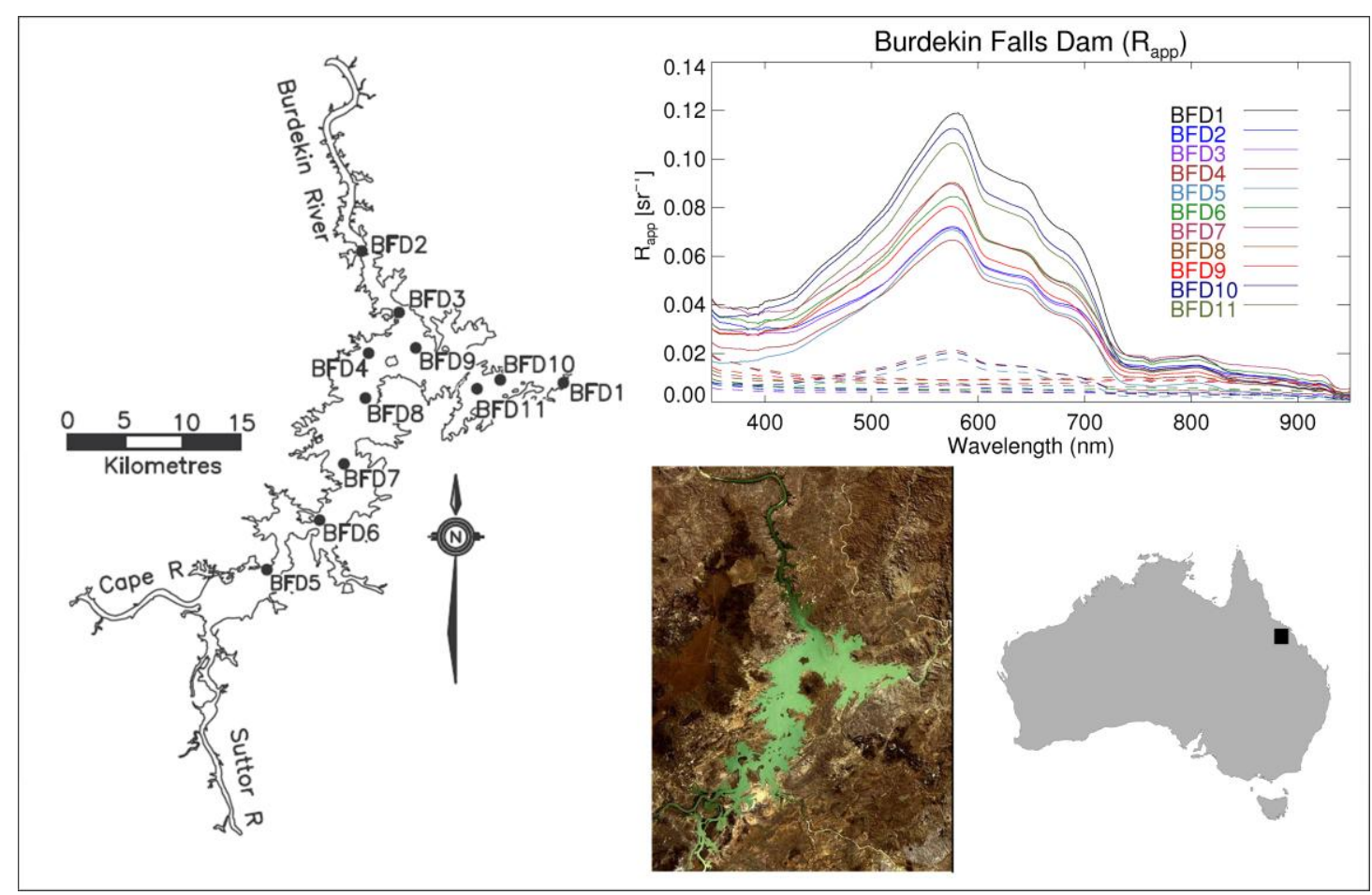

Figure 1 The mean $R_{a p p}$ spectra for the eleven stations at Burdekin Falls Dam. Dashed lines show the standard deviation of the measured spectra.

\subsection{Field Measurements}

During October 2008 the IOPs of the storage were measured at eleven stations (Figure 1). The backscattering properties were measured using a Hydroscat-6 backscattering sensor which emits light at six wavelengths and then measures the amount of light that is returned at a backscatter angle of $140^{\circ}$ (Maffione \& Dana 1997). For the standard method, simultaneous measurement of the absorption and attenuation of the water was required. Similarly, because the absorption of water is affected by the salinity and temperature of water (Pegau \& Zaneveld 1993) corrections must be made to the $a c-9$ absorption measurements before they can be used to correct the backscatter observations. These measurements were made using a Conductivity-Temperature-Depth (CTD) sensor. Due to the restricted craft size available for the field work it was not always practical to deploy the CTD, ac-9 and Hydroscat-6 instruments simultaneously within the water column. When the sampling craft was on station a small electric bilge pump was used to continuously sample the water from $0.5 \mathrm{~m}$ below the surface into a de-bubbling chamber. From there, it was gravity fed through a CTD sensor and then the $a c-9$. The $a c-9$ measured at nine wavelengths $(412,440,488,510,532,555,650,676$ and $715 \mathrm{~nm}$ ). The Hydroscat-6 was deployed directly into the lake at a depth of $0.5 \mathrm{~m}$. For the Burdekin Falls Dam measurements the Hydroscat-6 measured at wavelengths of 420 , $442,488,550,676$ and $852 \mathrm{~nm}$.

\section{In situ spectroradiometric data}

Two RAMSES spectroradiometers were mounted in a cage. One spectroradiometer was fitted with a cosine collector and was orientated upwards in the cage to measure the downwelling irradiance and one radiance collector was orientated downwards to measure the upwelling radiance. The cage was lowered on the unshaded side of the vessel to minimize the shading effects.
For each station of the 2008 field campaign, the simultaneous measurements of downwelling irradiance and upwelling radiance were combined to calculate the above surface reflectance. The observed reflectance spectra were convolved with the MERIS band response functions to produce simulated in situ spectra.

\subsection{Standard Backscattering Measurement}

\section{Temperature and Salinity Correction}

The effect of temperature and salinity on the absorption of water is linear, but wavelength dependent. The absorption co-efficient measured by the $a c-9$ was characterised by Pegau et al. (1997) as:

$$
a_{m}^{T S}(\lambda, T, S)=a_{m}\left(\lambda, T_{r}, 0\right)+\psi_{T}\left(T-T_{r}\right)+\psi_{S} S
$$

where $T$ is the temperature, $T_{r}$ is a reference temperature and $S$ is the salinity in Practical Salinity Units (PSU). The PSU of a sample of water is defined in terms of the ratio of electrical conductivity of the sample to that of a potassium chloride $(\mathrm{KCl})$ solution. The $\psi$ values are linear temperature $(\mathrm{T})$ and salinity (S) slopes. As the study site was a freshwater impoundment the effect of salinity was negligible and a correction was not applied. The values for the temperature slope were taken to be those reported by Pegau et al. (1997). The temperature effect is negligible except at the red and NIR wavelengths but as these values are used to correct scattering at all wavelengths there is an indirect effect on all wavelengths.

As the scattering coefficient is only affected by temperature or salinity in the ultraviolet wavelengths the effect on the attenuation coefficient in the visible and NIR regions is the same as the absorption coefficient (WET Labs Inc 2005). 


\section{Scattering Correction}

The dual beam spectrophotometer consists of two flow tubes to measure the attenuation and the absorption. In the nonreflecting attenuation flow tube, all photons that are scattered are absorbed by the sides of the tube, whereas the absorption tube is highly reflective so that those photons are reflected back into the detector at the end of the tube. This means that photons are only removed from the stream through absorption by the medium. In practice photons that are scattered backwards do not reach the detector, there are some photon losses due to the imperfect reflection of the flow tube and some photons reach the end of the tube but evade the detector (Kirk 1992; Mueller et al. 2003).

Zaneveld et al. (1994) suggested three scattering correction methods. The first assumes that the absorption in the near infrared (NIR) $(a(715))$ for the water quality parameters is negligible and the absorption is entirely due to pure water. The second approach assumes that the scattering error is a wavelength independent fraction $(\varepsilon)$ of the measured scattering coefficient and so calculates the value for the NIR and applies this value to all wavelengths. There is some confusion in the literature as to accepted values. Sources recommend that a value of $\varepsilon \approx 0.14$ be used for predominantly biological particles and $\varepsilon$ $\approx 0.18$ for waters dominated by suspended sediments (Mueller et al. 2003; WET Labs Inc 2005) citing work by Kirk (1992). However, Kirk's paper reports values for a tube reflectance of $94 \%$ and an acceptance angle for the detector of $180^{\circ}$ of $\varepsilon \approx 0.160$ be used for predominantly biological particles and $\varepsilon \approx$ 0.121 for waters dominated by suspended sediments. The values will vary with the design of the flow tube but since $\varepsilon$ is dependent on the scattering phase function of the medium it is unlikely that the relative proportions should change. The third method negates the need to assume an a priori scattering error and calculates the proportional error in the NIR and applies that to all wavelengths. The correction becomes:

$$
a(\lambda)-a_{w}(\lambda)=a_{m}^{T S}(\lambda)-\left[\frac{a_{m}^{T S}\left(\lambda_{N R}\right)}{c_{m}^{T S}\left(\lambda_{N R}\right)-a_{m}^{T S}\left(\lambda_{N R}\right)}\right]\left[c_{m}^{T S}(\lambda)-a_{m}^{T S}(\lambda)\right]
$$

This method still assumes that the absorption in the NIR $(a(715))$ for the water quality parameters is negligible, but allows for the scattering correction to change with wavelength and the material present. The first method is attractive due to its simplicity but the third method, while requiring simultaneous measurement of absorption and attenuation, should be the most accurate. The third method was applied in this case.

\section{Correction for Raw Hydroscat-6 Data}

In high light-attenuating waters the measured backscatter can be appreciably lower than the true backscatter. This effect can be corrected by:

$$
b_{b}(\lambda)=\sigma\left(K_{b b}\right) b_{b u}(\lambda) \quad(\text { Dana } \& \text { Maffione 2002) }
$$

where $b_{b}$ and $b_{b u}$ are the corrected and uncorrected backscatter respectively.

The factor $\sigma$ is calculated by:

$$
\sigma\left(K_{b b}\right)=k_{1} \exp \left(k_{\exp } K_{b b}\right)
$$

where $K_{b b}=a+0.4 b$ and with $k_{1}$ set by calibration so the value $\sigma\left(K_{b b w}\right)=1$ where $K_{b b w}$ is the attenuation, excluding that of pure water, of the water in which the instrument was calibrated (HOBILabs Inc 2008).

\subsection{Alternative Backscattering Measurement}

The alternative backscattering measurement used in this paper is a slightly modified version of the method referred to as the simulation method in Ma et al. (2009). In that paper the simulation method was used to provide validation data for a proposed alternative to the sigma correction described above. As that technique has further complications that will not be discussed here, only the simulation method will be considered. The two modifications to the simulation method were the source of the absorption values and the reflectance model.

The original paper sourced the absorption from laboratory measurements. In this paper the absorption values are sourced from the $a c-9$. The $a c-9$ measurements were used for corrections in the standard backscattering measurement. A single absorption measurement source was considered desirable so as to minimise spurious differences due to the source of the absorption measurements.

In Ma et al. (2009) the model that is used is the linear form of the Gordon, Brown and Jacobs (1975) semi-analytical model for in-water reflectance.

$$
R\left(\lambda, 0^{-}\right)=\sum_{n=0}^{n=3} f_{n}(\lambda)\left[\omega_{b}(\lambda)\right]^{n}, \omega_{b}(\lambda)=\frac{b_{b}(\lambda)}{a(\lambda)+b_{b}(\lambda)}
$$

The proportionality factor $f$ is often referred to as the anisotropy factor as it represents a correction for the direction distribution of light in the upwelling and downwelling fields. Gordon et al. (1988) stated that the error from leaving out the second order term is approximately $\frac{\omega_{b}}{1+\omega_{b}}$ Observations for Lake Taihu previously reported in Ma et al. (2006) (see Figure 9) showed $\omega_{\mathrm{b}}$ ranges from $0.1-0.6$ which equates to a $9 \%-37 \%$ error. The range of $\omega_{\mathrm{b}}$ for Burdekin Falls Dam was similar, so the second order term was retained in the reflectance model.

$$
\begin{aligned}
R_{r s}(\lambda)=\frac{R\left(0^{-}, \lambda\right)}{Q} & =\frac{f_{1}}{Q} \frac{b_{b}}{a+b_{b}}+\frac{f_{2}}{Q}\left(\frac{b_{b}}{a+b_{b}}\right)^{2} \\
& =f_{1 Q} \frac{b_{b}}{a+b_{b}}+f_{2 Q}\left(\frac{b_{b}}{a+b_{b}}\right)^{2}
\end{aligned}
$$

where $Q$ is the ratio of upwelling irradiance to upwelling radiance. The conversion factor from radiance to irradiance changes in a reasonably parallel manner to changes in $f$ so that the $f_{Q}$ factors can be treated as spectrally invariant (Morel \& Gentili 1993). Since reflectance is affected by the angular structure of the light within the water then the values should be affected by the solar altitude, the scattering phase function and proportion of diffuse light (Kirk 1984). There is no guarantee that the $f_{Q}$ factors will be constant within the same water body and under differing illumination conditions, so ideally they should be allowed to vary in the particle backscatter retrieval. If the particulate backscattering model and the measured absorption are substituted into this expression the remote sensing reflectance becomes a function of four unknowns. 


$$
R_{r s}(\lambda)=f_{1 Q} \frac{b_{b p o}\left(\frac{\lambda}{\lambda_{0}}\right)^{\gamma}+b_{b w}}{a+b_{b p o}\left(\frac{\lambda}{\lambda_{0}}\right)^{\gamma}+b_{b w}}+f_{2 Q}\left(\frac{b_{b p o}\left(\frac{\lambda}{\lambda_{0}}\right)^{\gamma}+b_{b w}}{a+b_{b p o}\left(\frac{\lambda}{\lambda_{0}}\right)^{\gamma}+b_{b w}}\right)^{2}
$$

For each station with spectroradiometric observations, simultaneous measurements of downwelling irradiance $\left(E_{d}\right)$ and upwelling radiance $\left(L_{u}\right)$ were combined to calculate the above surface reflectance $\left(R_{a p p}\right)$. These observations were convolved with the Gaussian bands with a FWHM of $10 \mathrm{~nm}$ (WET Labs Inc 2006) centred on the $a c-9$ wavelengths to create a set of over-determined simultaneous equations. These non-linear equations can be solved iteratively using non-linear least squares fitting. Using initial estimates of the unknown parameters the system of equations are manipulated to form the matrix equation:

$\mathbf{A} \partial p=\partial \beta$

where

$$
\mathbf{A}=\left[\begin{array}{cccc}
\frac{\partial R_{a p p}}{\partial f_{1 Q}}\left(\lambda_{1}\right) & \frac{\partial R_{a p p}}{\partial f_{2 Q}}\left(\lambda_{1}\right) & \frac{\partial R_{a p p}}{\partial b_{b p 0}}\left(\lambda_{1}\right) & \frac{\partial R_{a p p}}{\partial \gamma}\left(\lambda_{1}\right) \\
\cdot & \cdot & \cdot \\
\cdot & \cdot & \cdot \\
\frac{\partial R_{a p p}}{\partial f_{1 Q}}\left(\lambda_{9}\right) & \frac{\partial R_{a p p}}{\partial f_{2 Q}}\left(\lambda_{9}\right) & \frac{\partial R_{a p p}}{\partial b_{b p 0}}\left(\lambda_{9}\right) & \frac{\partial R_{a p p}}{\partial \gamma}\left(\lambda_{9}\right)
\end{array}\right]
$$

is a matrix of partial derivatives

$\partial p=\left[\begin{array}{c}\partial f_{1 Q} \\ \partial f_{2 Q} \\ \partial b_{b p 0} \\ \partial \gamma\end{array}\right]$

is a matrix of the desired corrections to the parameter estimates and

$\partial \beta=\left[\begin{array}{c}R_{\text {meas }}\left(\lambda_{1}\right)-R\left(f_{Q}, \partial b_{b p 0}, \partial \gamma, a\left(\lambda_{1}\right), \lambda_{1}\right) \\ \cdot \\ \cdot \\ \cdot \\ \cdot \\ R_{\text {meas }}\left(\lambda_{9}\right)-R\left(f_{Q}, \partial b_{b p 0}, \partial \gamma,\left(\lambda_{9}\right), \lambda_{9}\right)\end{array}\right]$

is a matrix of the difference between the measured and forward estimated reflectances.

The standard solution of this expression is

$$
\partial p=\left[\mathbf{A}^{\mathbf{T}} \mathbf{A}\right]^{-1} \mathbf{A}^{\mathbf{T}} \partial \beta
$$

It is possible to differentially weight the importance of each band to give greater influence to those bands which are deemed to be more reliable. In this case the weight matrix is a square (9x9) diagonal matrix $(\mathrm{W})$ where $\mathrm{W}_{\mathrm{ii}}=$ signal to noise ratio of band $i$.

The solution then becomes

$$
\partial p=\left[\mathbf{A}^{\mathrm{T}} \mathbf{W A}\right]^{-\mathbf{1}} \mathbf{A}^{\mathrm{T}} \mathbf{W} \partial \beta
$$

\section{RESULTS}

\subsection{Standard Backscattering Measurement}

The standard backscattering measurement method resulted in measurements of $b_{b p 0}$ with a range of $0.22-0.53 \mathrm{~m}^{-1}$ and $\gamma$ with a range of -1.14 to $-1.57 \mathrm{~nm}^{-1}$. More detailed results can be found in Campbell et al. (2011).

\subsection{Alternative Backscattering Measurement}

The method was applied to the mean spectra measured at each of the stations with the bands being weighted in inverse proportion to the standard deviation of that measurement. When this iterative approach is used to extend weighted least squares to cover non-linear problems there is the risk that the non-linear matrix inversion method will find a local minimum dependent on the starting point, rather than the absolute minimum (Keller 2001). As the results in this case were highly dependent on the starting condition it would appear that this was occurring. In an attempt to circumvent this ill-posed problem an optimisation approach was employed which used a forward model to calculate a reflectance spectrum from water quality constituent values and then used a similarity measure to match it to the measured reflectance spectrum was employed.

\section{Particle Swarm Optimisation}

The PSO is the stochastic search technique which includes a random element in the search approach that was first applied to ocean colour by Slade et al. (2004). The algorithm represents the solution as an $n$-dimensional vector in an $n$-dimensional solution space. It then mimics the action of a swarm by generating a number of potential solutions or 'particles' and after each iteration having them react to the closest match in its local area as well as the best match from all the particles. The best match can be defined by any appropriate cost function or similarity measure.

Let $\mathbf{x}_{\mathbf{j}}$ be a particle and the position of $\mathbf{x}_{\mathbf{j}}$ after the next iteration as $\mathbf{x}_{\mathbf{j}}+\boldsymbol{\Delta} \mathbf{x}_{\mathbf{j}}$ where $\Delta \mathbf{x}_{\mathbf{j}}$ is referred to as the trajectory. The trajectory is related to the value of two vectors, the vector connecting $\mathbf{x}_{\mathbf{j}}$ to the best match that it has previously made $\left(\mathbf{x}_{\mathbf{j}, \text { best }}\right)$ and the vector connecting $\mathbf{x}_{\mathbf{j}}$ to the best match that any of the particles have made ( $\mathbf{x}_{\mathbf{G}, \text { best }}$ ). The random element is introduced by generating random number multiples of the

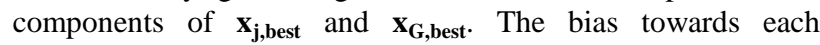
component vector is controlled by two weight constants $c_{l}$ and $c_{2}$. To aid in the convergence the sum of the vectors is multiplied by a constriction factor $\chi$.

Formally,

$$
\begin{aligned}
& \mathbf{x}_{\mathbf{j}}(t+1)=\mathbf{x}_{\mathbf{j}}(t)+\Delta \mathbf{x}_{\mathbf{j}}(t+1) \\
& \Delta \mathbf{x}_{\mathbf{j}}(t+1)=\chi\left(\Delta \mathbf{x}_{\mathbf{j}}(t)+\Phi_{1}\left(\mathbf{x}_{\mathbf{j}, \text { best }}-\mathbf{x}_{\mathbf{j}}(t)\right)+\Phi_{2}\left(\mathbf{x}_{\mathbf{G}, \text { best }}-\mathbf{x}_{\mathbf{j}}(t)\right)\right)
\end{aligned}
$$

In this case the search space is three dimensional so 
$\Phi_{m}=c_{m}\left[\begin{array}{ccc}r_{m, 1} & 0 & 0 \\ 0 & r_{m, 2} & 0 \\ 0 & 0 & r_{m, 3}\end{array}\right]$

where $r_{m, i}$ are random scalars

uniformly distributed between 0 and 1 .

$$
\chi=\frac{2}{\left|2-\phi-\sqrt{\phi^{2}-4 \phi}\right|} \quad \phi=c_{1}+c_{2}, \quad \phi>4
$$

The $\phi>4$ restriction is required to prevent the values of the trajectories from becoming cyclical and hence not randomly searching the solution space (Clerc \& Kennedy 2002). The parameters $c_{1}$ and $c_{2}$ were set at 2.05 (Slade et al. 2004).

The PSO was applied with 64 particles to the reflectance spectra allowing the four unknowns to vary and using the minimum Euclidian distance as the similarity measure. The solutions for the two anisotropy factors were limited to physically meaningful positive numbers. Once again the calculation did not supply a unique solution. It has been shown previously that the inverse problem of retrieving scattering and absorption properties for ocean colour using the case-I water simplification is ill-posed or ambiguous in that many disparate combinations of IOPs can produce the same reflectance spectrum (Defoin-Platel \& Chami 2007). Furthermore, it was shown that the ambiguity error on the total backscattering co-efficient was higher in turbid scattering waters. To see whether this was true in this case the PSO was run 20 times on the same spectrum. The results for $\mathrm{b}_{\mathrm{bp} 0}$ and $\gamma$ are plotted in Figure 2.

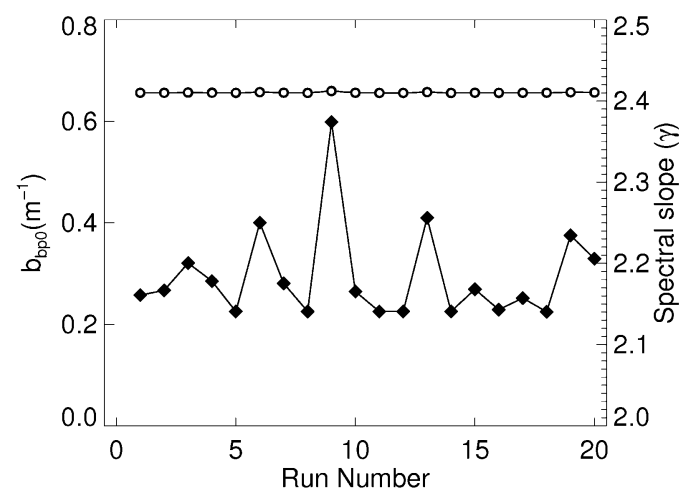

Figure 2 The magnitude of the retrieved bbp0 (closed diamonds) and spectral slope $(\gamma)$ (open circles) values for the 20 runs.

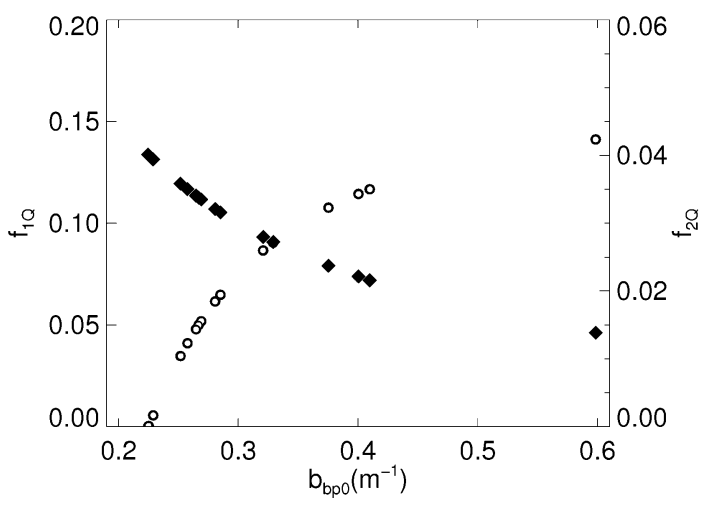

Figure 3 The correlation between the retrieved values of values $\mathrm{b}_{\mathrm{bp} 0}, \mathrm{f}_{1 \mathrm{Q}}$ (closed diamonds) and $\mathrm{f}_{2 \mathrm{Q}}$ (open circles) for the 20 runs.
It is clear from Figure 2 that very similar reflectance spectra can be produced even though there are large differences in the value of $b_{b p 0}$. This happens because the values for the anisotropy factors are allowed to vary independently to $b_{b p 0}$ and $\gamma$. When the factors are allowed to vary independently the values in the best fit solution are clearly correlated as seen in Figure 3.

Notwithstanding the previous discussion on the variability of the $f_{Q}$ factors and their controls, to resolve the ambiguity it was necessary to fix the values of the anisotropy factors. Using the large number of reflectance spectra that were created by using a precise numerical model, Hydrolight, Lee et al. (1999) assert that for case-II coastal water values $\mathrm{f}_{1 \mathrm{Q}}=0.084$ and $\mathrm{f}_{2 \mathrm{Q}}=0.17$ can be used. The resulting $b_{b p 0}$ and $\gamma$ values are plotted against the same values measured using the more typical Hydroscat- 6 approach in Figure 4 and Figure 5. The reflectance method underestimates by approximately $50 \%$ in the case of $b_{b p 0}$ and in the case of $\gamma$ it has an appreciable bias and overestimates by about $40 \%$. The apparent linear trend in both plots suggests that better a priori values for the $f_{Q}$ factors would lead to more consistent results, however since this requires better information on the scattering phase function the problem becomes circular and a case of petitio principii.

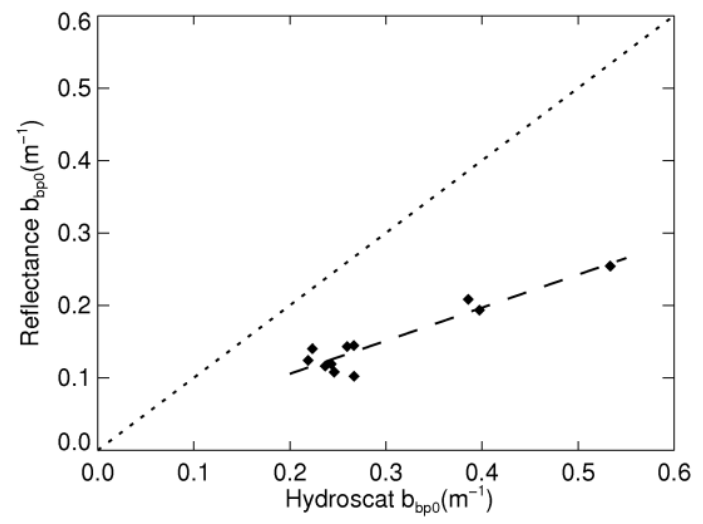

Figure 4 The Hydroscat- 6 derived $b_{\mathrm{bp} 0}$ vs Reflectance derived $\mathrm{bb}_{\mathrm{p} 0}$ for the Burdekin Falls Dam stations. The dashed line shows a line of best fit for the data and the dotted line is a $1: 1$ correspondence line.

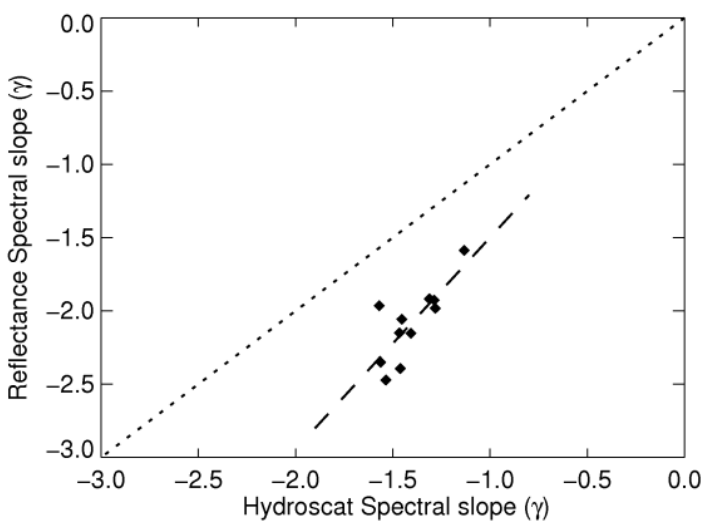

Figure 5 The Hydroscat- 6 derived $\gamma$ vs. Reflectance derived $\gamma$ for the Burdekin Falls Dam stations. The dashed line shows a line of best fit for the data and the dotted line is a 1:1 correspondence line. 


\section{CONCLUSIONS}

When compared to the conventional corrected Hydroscat-6 observations for the data sets from Burdekin Falls Dam, it was found that the reflectance method underestimated $b_{\mathrm{bp} 0}(532)$ by approximately $50 \%$ and overestimated $\gamma$ by approximately $40 \%$. Differences of this scale in specific backscattering spectra will have a pronounced effect on the parameterisation and subsequent performance of semi-analytical water quality parameter retrieval algorithms. This has implications for any water quality monitoring regimes that rely on semi-analytical algorithms.

\section{ACKNOWLEDGEMENTS}

The authors would like to thank Ms Lesley Clementson of CSIRO for the laboratory measurements and Dr Arnold Dekker and Dr Vittorio Brando. We would like to express their gratitude to the water body operator Sunwater for their assistance in the field operations.

\section{REFERENCES}

Campbell, G, Phinn, SR \& Daniel, P 2011, 'The specific inherent optical properties of three sub-tropical and tropical water reservoirs in Queensland, Australia.' Hydrobiologia, vol. 658 , no. 1 , pp. 233-52.

Clerc, M \& Kennedy, J 2002, 'The particle swarm - explosion, stability, and convergence in a multidimensional complex space', IEEE Transactions on Evolutionary Computation, vol. 6, no. 1 , pp. 58-73.

Dana, DR \& Maffione, RA 2002, 'Determining the backward scattering coefficient with fixed-angle backscattering sensorsrevisited', in Ocean Optics XVI, Santa Fe, NM., p. 9.

Defoin-Platel, M \& Chami, M 2007, 'How ambiguous is the inverse problem of ocean color in coastal waters?' Journal of Geophysical Research-Oceans, vol. 112, no. C3, p. C03004.

Gordon, HR, Brown, OB, Evans, RH, Brown, JW, Smith, RC, Baker, KS \& Clark, DK 1988, 'A Semianalytic radiance model of ocean color', Journal of Geophysical Research, vol. 93, no. D9, pp. 10909-24.

Gordon, HR, Brown, OB \& Jacobs, MM 1975, 'Computed relationships between the inherent and apparent optical properties of a flat homogeneous ocean', Applied Optics, vol. 14 , no. 2 , pp. 417-27.

HOBILabs Inc 2008, HydroScat-6 Spectral Backscattering Sensor User's manual (Revision H), Hydro-Optics, Biology, and Instrumentation Laboratories, Inc. (HOBILabs).

Keller, PA 2001, 'Comparison of two inversion techniques of a semi-analytical model for the determination of lake water constituents using imaging spectrometry data', The Science of The Total Environment, vol. 268, no. 1-3, pp. 189-96.

Kirk, JTO 1984, 'Dependence of relationship between inherent and apparent optical properties of water on solar altitude', Limnology and Oceanography, vol. 29, no. 2, pp. 350-6.
1992, 'Monte Carlo modeling of the performance of a reflective tube absorption meter', Applied Optics, vol. 31, no. 30, pp. 6463-8.

Lee, Z, Carder, KL, Mobley, CD, Steward, RG \& Patch, JS 1999, 'Hyperspectral remote sensing for shallow waters. 2. Deriving bottom depths and water properties by optimization', Applied Optics, vol. 38, no. 18, pp. 3831-43.

Ma, RH, Pan, DL, Duan, HT \& Song, QJ 2009, 'Absorption and scattering properties of water body in Taihu Lake, China: backscattering', International Journal of Remote Sensing, vol. 30, no. 9, pp. 2321-35.

Ma, RH, Tang, J \& Dai, J 2006, 'Bio-optical model with optimal parameter suitable for Taihu Lake in water colour remote sensing', International Journal of Remote Sensing, vol. 27 , no. 19 , pp. $4305-28$.

Maffione, RA \& Dana, DR 1997, 'Instruments and methods for measuring the backward-scattering coefficient of ocean waters', Applied Optics, vol. 36, no. 24, pp. 6057-67.

Morel, A \& Gentili, B 1993, 'Diffuse reflectance of oceanic waters. 2. Bidirectional aspects', Applied Optics, vol. 32, no. 33, pp. 6864-72.

Mueller, JL, Fargion, GS, McClain, CR, Pegau, S, Zaneveld, JRV, Mitchell, BG, Kahru, M, Wieland, J \& Stramska, M 2003, Ocean Optics Protocols For Satellite Ocean Color Sensor Validation, Revision 4, Volume IV: Inherent Optical Properties: Instruments, Characterizations, Field Measurements and Data Analysis Protocols, NASA, Greenbelt, Maryland.

Pegau, WS, Gray, D \& Zaneveld, JRV 1997, 'Absorption and attenuation of visible and near-infrared light in water: dependence on temperature and salinity', Applied Optics, vol. 36, no. 24, pp. 6035-46.

Pegau, WS \& Zaneveld, JRV 1993, 'Temperature-Dependent Absorption of Water in the Red and Near-Infrared Portions of the Spectrum', Limnology and Oceanography, vol. 38, no. 1, pp. 188-92.

Slade, WH, Ressom, HW, Musavi, MT \& Miller, RL 2004, 'Inversion of ocean color observations using particle swarm optimization', IEEE Transactions on Geoscience and Remote Sensing, vol. 42, no. 9, pp. 1915-23.

WET Labs Inc 2005, ac-9 Protocol Document (Revision J), Western Environmental Technology Laboratories (WETLabs), Philomath, OR.

2006, Absorption and Attenuation Meter ac-9 User's Guide (Revision O), Western Environmental Technology Laboratories (WETLabs), Philomath, OR.

Wu, G, Cui, L, Duan, H, Fei, T \& Liu, Y 2011, 'Absorption, backscattering coefficients and their relations with water constituents of Poyang Lake, China', Applied Optics, no. Doc. ID 148611 (posted 12 October 2011, in press).

Zaneveld, JRV, Kitchen, JC \& Moore, C 1994, 'The scattering error correction of reflecting-tube absorption meters.' in Ocean Optics XII, vol. 2258, pp. 44-55. 